
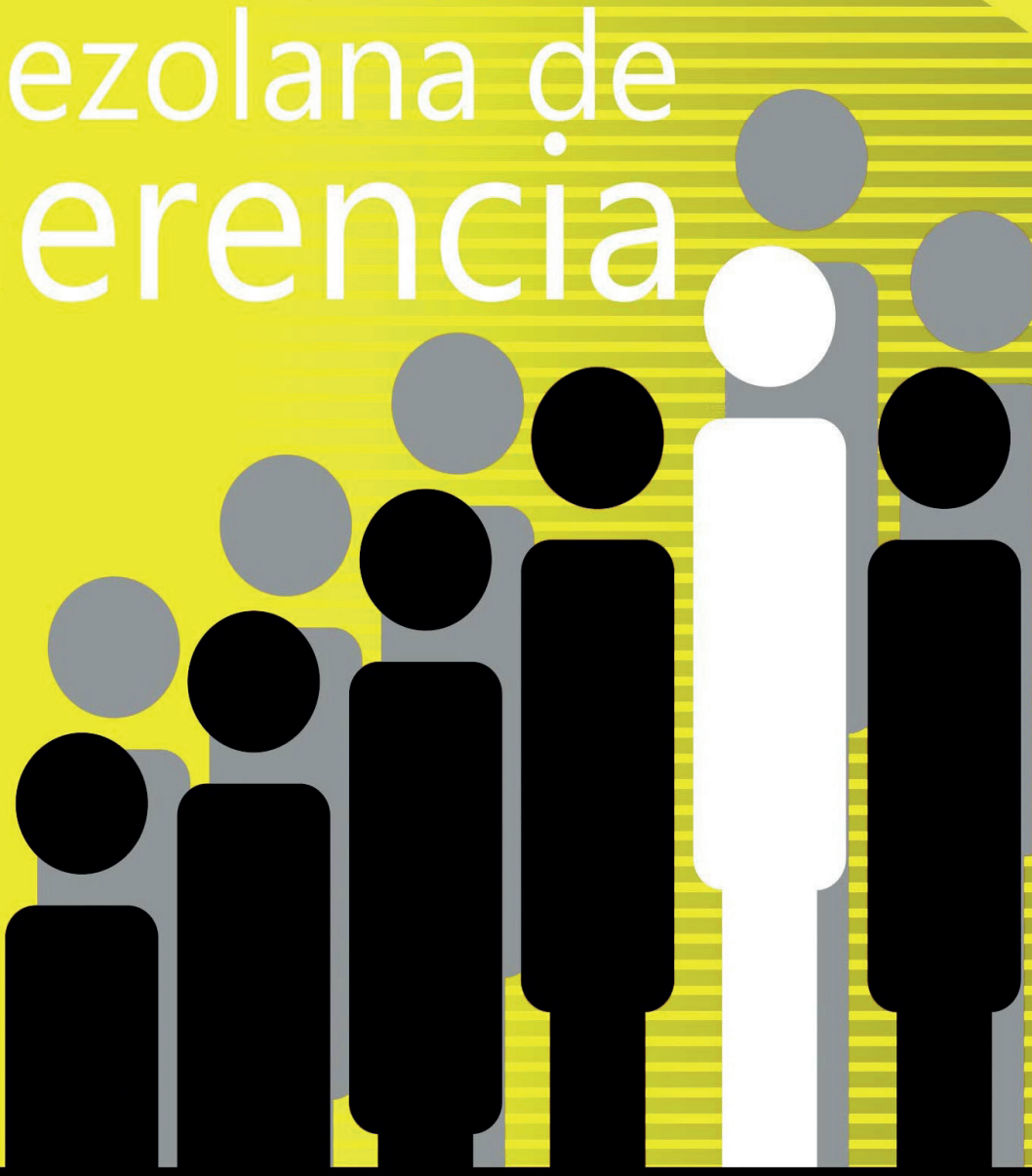


\title{
Inteligencia artificial (IA) aplicada a la gestión pública
}

\author{
Ocaña-Fernández, Yolvi ${ }^{\star}$ \\ Valenzuela-Fernández, Luis Alex ${ }^{*}$ \\ Vera-Flores, Miguel Angel ${ }^{* \star \star}$ \\ Rengifo-Lozano, Raúl Alberto ${ }^{* * *}$
}

\section{Resumen}

La implementación de sistemas basados en inteligencia artificial (IA) ha pasado la barrera del campo académico y debido a sus potencialidades se viene desarrollando en otros campos tales como la gestión pública por lo que urge la necesidad de tener un panorama actualizado al respecto. Este artículo tiene como objetivo abordar el análisis de la IA destacando su trascendencia en el campo de la gestión, administración pública y gobierno, resaltando las oportunidades significativas, la evaluación de impactos y el potencial que plantea la IA. La presente revisión ofrece una visión panorámica y significativa acerca de la IA y su impacto en el campo de la gestión y administración pública, acerca de sus logros, así como sensibles controversias. Finalmente se muestran las oportunidades y desafíos críticos de aplicación de la IA en el sector público.

Palabras clave: inteligencia artificial; gestión pública; gobernanza digital; administración pública.

\section{Recibido: 15.12 .20 Aceptado: 20.02 .21}

* Docente investigador de la Universidad Privada San Juan Bautista, Lima, Perú, Doctor en Educación. E-mail: yolvi.ocana@upsjb.edu.pe. ORCID: https://orcid.org/0000-0002-2566-6875

** Maestro en Docencia Universitaria, Biólogo y docente de Biología para las ciencias e ingenierías en la Escuela de Estudios Generales de la Universidad Nacional Mayor de San Marcos. Docente investigador reconocido por Renacyt. Correo: Ivalenzelaf@unmsm.edu.pe. Orcid: http://orcid.org/0000-0002-8743-4092.

*** Docente de la universidad Nacional Mayor de San Marcos, doctorado en medicina, con amplia experiencia en gestión pública. E-mail: mavera290@gmail.com, Orcid 0000-0001-8970-751X

*** Ph.D. en Administración, Doctor en Política Fiscal y Sistema Tributario, Doctor en Economía. Docente de la Universidad Nacional Mayor de San Marcos - Perú. E-mail: rrengifolo@unmsm.edu.pe. ORCID: https:// orcid.org/0000-0002-6545-6442. 


\title{
Artificial intelligence (AI) applied to public management
}

\begin{abstract}
The implementation of systems based on artificial intelligence (AI) has passed the barrier of the academic field and due to its potentialities it has been developing in other fields such as public management, so there is an urgent need to have an updated panorama in this regard. This article aims to address the analysis of Al highlighting its significance in the field of management, public administration and government, highlighting the significant opportunities, impact assessment and potential that Al poses. The present review offers a panoramic and significant vision about $\mathrm{Al}$ and its impact in the field of public management and administration, about its achievements, as well as sensitive controversies. Finally, the critical opportunities and challenges of applying $\mathrm{Al}$ in the public sector are shown.
\end{abstract}

Key words: artificial intelligence; public management; digital governance; public administration.

\section{Introducción}

Establecida como una disciplina académica en la década de 1950, la inteligencia artificial (IA) siguió siendo un área de relativa oscuridad científica y de interés práctico limitado durante más de medio siglo. La IA, como idea, apareció por primera vez poco después de que los humanos desarrollaran la informática digital electrónica y desde entonces ha enfrentado oleadas de publicidad y pesimismo (Press, 2017). De acuerdo con Chui (2017) la IA se refiere a la capacidad de las máquinas para exhibir una inteligencia similar a la humana, por ejemplo, resolver un problema independientemente de algún software manufacturado. El ritmo vertiginoso del desarrollo en IA está brindando oportunidades sin precedentes (Park, 2017; Issa, Sun \& Vasarhelyi, 2016), las que según Abduljabbar, Dia, Liyanage,
\& Bagloee (2019) las innovaciones introducidas por la IA incluyen métodos computacionales muy avanzados que imitan la forma en que funciona el cerebro humano.

En la actualidad, debido al auge de disciplinas científicas derivadas de la aplicación de la IA tales como big data (almacenamiento y manejo de enormes volúmenes de datos), machine learning (generación de sistemas de aprendizaje automático), deep learning (empleo de redes neuronales artificiales de niveles jerárquicos), han ingresado en el entorno de la gestión y administración pública (Haenlein \& Kaplan, 2019). De acuerdo con Dwivedi et al, (2019) los nuevos avances en el aprendizaje automático algorítmico y la toma de decisiones autónoma, lo que genera nuevas oportunidades para la innovación continua. La IA está lista para desencadenar la próxima ola de 
Yolvi Ocaña-Fernández; Luis Alex Valenzuela-Fernández; Miguel Angel Vera-Flores; Raúl Alberto Rengifo-Lozano

Inteligencia artificial aplicada a la gestión pública

disrupción digital, y las instituciones, organizaciones y/o empresas deben hallarse preparadas para ello ahora (Chui, 2017; Nadikattu, 2016). Según Helbing et al, (2019) la IA ofrece un enorme potencial transformador para el aumento y la sustitución potencial de tareas y actividades humanas dentro de una amplia gama de aplicaciones industriales, intelectuales y sociales.

Si bien los beneficios de mayores niveles de adopción de IA en muchos sectores de la economía global se sienten en el contexto de una mayor eficiencia, productividad mejorada y confiabilidad, dicho contexto de innovación positiva no es del todo universalmente bienvenida o aceptada a nivel mundial; ya que según lo planteado por se tiene que las estimaciones para el desplazamiento laboral producto de la automatización apuntan que hasta un tercio de las actividades laborales presentes hoy en día, podrían verse afectadas hacia el 2030 (Dwivedi et al, 2019).

Según lo manifestado por Dwivedi et al, (2019) la IA es un concepto que ha formado parte del discurso público durante décadas, a menudo representado en películas de ciencia ficción o debates de corte apocalíptico donde máquinas inteligentes se apoderarán del mundo y relegan a la raza humana a una existencia servil mundana en apoyo del nuevo orden impuesto por la IA. Si bien dicha representación tiene un matiz de caricaturesco, la realidad es que la IA ya está presente y muchos de nosotros interactuamos regularmente con dicha tecnología en nuestra vida cotidiana. Habrá de tenerse muy en cuenta lo manifestado por Cath et al, (2018) sobre la IA, la cual según los citados autores no es meramente otra utilidad que necesita ser regulada solo una vez que esté madura; ya que en sí misma es una fuerza poderosa que está remodelando nuestras vidas, nuestras interacciones y nuestro entorno.

Con la finalidad de poder dimensionar el panorama referente a la IA y sus metodologías y su vinculación en la gestión pública, se ha procedido a realizar una colecta y depuración de trabajos científicos al respecto que abarquen tal problemática. Para ello se ha consultado las bases de datos EBSCO, Scopus, Medline, Taylor \& Francis, Scielo empleando como criterio de inclusión términos clave relativos a gestión y administración pública y IA para compilar y depurar información relevante con un máximo de 5 años de antigüedad, ello debido a los cambios constantes que a los que la IA está afecta, con la finalidad de poder analizar con una mejor proximidad el panorama actual. Se han excluido aquellos trabajos que tuvieron un margen superior a 5 años y otros que se enfocaban en criterios de programación y algoritmos de big data (código) por ser netamente técnicos en su naturaleza.

\section{Inteligencia Artificial en el gobierno y sector público}

De acuerdo con Henman (2020) se tiene que las nuevas tecnologías digitales están cambiando rápidamente el panorama de la prestación de servicios públicos; ya que los dispositivos móviles combinados con aplicaciones llevan los servicios públicos en línea a cualquier lugar donde se encuentre el ciudadano. Las tecnologías en red y wi-fi permiten que el suministro de información y la recopilación de datos codificados geográficamente se integren con los datos administrativos tradicionales, creando conjuntos de big data para generar conocimiento sobre poblaciones 
e individuos, los mismos que pueden ser procesados por técnicas basadas en IA a fin de optimizar la toma de decisiones. Los procesos automatizados de toma de decisiones administrativas se están expandiendo, y la IA (a través del aprendizaje automático o machine learning) proporciona formas más matizadas de tomar decisiones en circunstancias complejas. De acuerdo con Mikhaylov, Esteve \& Campion (2018) muchas organizaciones del sector público están cada vez más interesadas en utilizar la ciencia de datos y las capacidades de la IA para implementar políticas y generar eficiencias en entornos de alta incertidumbre.

Habrá de tenerse en cuenta lo expuesto por Sun y Medaglia (2019) que, en el caso de China, en una fase incipiente, la exageración vinculada a la introducción de la IA en el sector público estuvo inevitablemente acompañada de cierto grado de incertidumbre, ya que la aplicación de tales tecnologías en dicho sector vendría a ser la continuación de la explosión de la IA en el sector privado. Este aspecto no es nada novedoso ya que fue el sector privado el primero en adoptar la IA para los casos de organización, planificación y dirección de sus procesos. Por otro lado, Duan et al, (2019), mencionó que la introducción de la IA en acciones gubernamentales posibilitará una amplia gama de oportunidades únicas. Así mismo remarcaron que muchos de ellos comienzan a destacarse en un número creciente de puntos de vista sobre la IA en el sector público.

Según lo manifestado por Agarwal (2018) los administradores públicos no se encuentran preparados para afrontar los desafíos que exige la transformación/ derivación de la aplicación de las nuevas tecnologías, en especial las relacionadas con el manejo de ingentes cantidades de información orientadas a la toma de decisiones las cuales se sustentan en IA. A pesar de que muchas estructuras y procesos gubernamentales actuales han evolucionado durante las últimas décadas, probablemente se volverán irrelevantes o caducas en un futuro cercano. Debido a lo expuesto existe una necesidad imperiosa de sentar las bases para que los gobiernos reconsideren cómo podrán servir mejor a sus electores.

El impacto transformador de la IA ha generado un interés académico significativo con estudios recientes que según Dwivedi et al, (2019) han pasado de investigar los impactos y consecuencias de la tecnología a las implicaciones de rendimiento de la IA así como la implementación de sistemas basados en IA en diversas esferas que abarcan múltiples campos como el económico, militar, administrativo, educación, médico, seguridad, gestión, entre otros. De acuerdo con el trabajo realizado por Sun y Medaglia (2019) utilizando un estudio de caso para analizar los desafíos de aplicar la IA en el sector público del contexto chino, en base al análisis de tres grupos de partes interesadas: formuladores de políticas gubernamentales, gerentes/médicos de hospitales y gerentes de empresas en tecnologías de la información; con la finalidad de identificar cómo son percibidos los desafíos al adoptar la IA en el sector público en relación a los cambios y el impacto en los ciudadanos en el contexto de los desafíos políticos, legales y de políticas, así como amenazas a la seguridad nacional de empresas de propiedad extranjera. Los resultados generados mostraron que las diferentes partes interesadas tienen encuadres diversos $\mathrm{y}$, a veces, 
Yolvi Ocaña-Fernández; Luis Alex Valenzuela-Fernández; Miguel Angel Vera-Flores; Raúl Alberto Rengifo-Lozano

Inteligencia artificial aplicada a la gestión pública

contradictorios de los desafíos.

El razonamiento planteado por Shava \& Hofisi (2017), sustentado en los avances tecnológicos, se basó en el hecho de que la IA ya está generando un efecto importante en el valor del trabajo, y para los principales segmentos de la población, el valor humano ahora se determina por el costo de la inteligencia artificial equivalente, por lo tanto, la administración pública tendrá que responder a esta realidad. La introducción de la IA en el sector público abre nuevos escenarios tanto para los profesionales como para los investigadores. Un ejemplo particular, es el caso de China y Estados Unidos quienes han reconocido el valor de la IA para el sector público y su competitividad en la economía global (Wirtz, Weyerer, \& Geyer, 2019). Ser capaz de comprender y actuar en estos escenarios se vuelve ahora de suma importancia (Dwivedi et al, 2019). De acuerdo con Galloway \& Swiatek (2018) la IA puede ayudar a los profesionales de relaciones públicas a lidiar con las emociones de las personas mientras planean implementaciones. La IA ofrece grandes oportunidades para la administración pública, incluida la automatización de los procesos de flujo de trabajo, un procesamiento de información más rápido, una calidad de servicio mejorada o una mayor eficiencia laboral (Zheng et al, 2018).

Una forma en que la IA está comenzando a aplicarse en el gobierno local es mediante la introducción de análisis predictivo y tecnologías de apoyo a la toma de decisiones (Vogl, Seidelin, Ganesh \& Bright, 2019). Las aplicaciones de la IA podrían hacer que el gobierno trabaje de manera más eficiente y a la par que deja tiempo libre para que los empleados construyan mejores relaciones con los ciudadanos.
Dado que la satisfacción de los ciudadanos con las ofertas del gobierno digital deja mucho que desear, la IA puede ser una forma de cerrar la brecha al tiempo que mejora la participación ciudadana y la prestación de servicios (Mehr, Ash \& Fellow, 2017). Las IA tales como el machine learning, los sistemas basados en reglas, el procesamiento del lenguaje natural y el reconocimiento de voz, cuando son adoptados en el sector público, tienen implicaciones potenciales para diversos aspectos del accionar gubernamental, incluyendo el funcionamiento interno de las agencias gubernamentales, la relación entre los gobiernos y ciudadanos, y el papel de los gobiernos como reguladores (Dwivedi et al, 2019).

El estudio expuesto por Abbasi \& El Hanandeh (2016) acerca de la aplicación concreta de la IA en un rubro referido a la administración pública sobre poder garantizar una gestión adecuada de los residuos sólidos urbanos (RSU) en una localidad australiana. En dicho proceso implementaron sistemas avanzados de pronóstico basados en IA. En dicho estudio, se compararon cuatro algoritmos de IA por su precisión para predecir las tasas de generación de desechos. Los resultados mostraron que los modelos de IA brindanron herramientas prometedoras que pueden permitir a los administradores una mejor toma de decisiones para pronosticar con éxito las tendencias futuras en la gestión de RSU, así como en la planificación y el diseño de la gestión de RSU. Según los avances desarrollados en algunos aspectos relacionados a la administración pública la implementación y desarrollo de procesos basados en IA han demostrado superioridad a los modelos convencionales de gestión y manejo de RSU. 
Otro ejemplo relevante es el trabajo desarrollado por Kouziokas (2017) sobre las mejoras en la calidad y seguridad de los servicios de transporte público en Grecia, donde mencionó que la administración pública ha optado por aplicar métodos de la IA para generar modelos predictivos de redes neuronales artificiales en las áreas de transporte de alto riesgo de delincuencia, además de sistemas de información geográfica para realizar análisis espaciales con el fin de identificar las regiones con una alta concentración de incidentes delictivos. Dicho estudio implementó un algoritmo de gradiente conjugado escalado, como algoritmo de entrenamiento, para la generación de los modelos de redes neuronales de retroalimentación, ya que el mismo fue considerado como uno de los algoritmos de aprendizaje más rápidos en comparación con varios otros equivalentes. Sus resultados mostraron una muy buena precisión de predicción, considerándolo como muy prometedor y puede promover políticas de gestión del transporte más seguras, especialmente en las ciudades donde las tasas de criminalidad son muy altas.

Otro logro destacado es el trabajo desarrollado por Abduljabbar et al, (2019) sobre la aplicación de la IA en el campo del transporte con el objetivo superar los desafíos de la creciente demanda de viajes, las emisiones de dióxido de carbono, problemas de seguridad y la degradación ambiental. Los problemas de transporte se convierten en un desafío cuando el sistema y el comportamiento de los usuarios es demasiado difícil de modelar y predecir los patrones de viaje. Valiéndose para ello de una gran cantidad de datos cuantitativos y cualitativos de las características y variables del sistema de transporte $y$ métodos basados en IA tales como redes neuronales artificiales, algoritmos genéticos, entre otros. Los resultados del análisis mostraron que la IA se puede emplear de forma conveniente para resolver el desafío de aumentar la demanda de viajes, las emisiones de $\mathrm{CO}_{2}$, los problemas de seguridad y el desperdicio de combustibles.

Según los citados investigadores la próxima aplicación prometedora de IA en el transporte serán los vehículos conectados y autónomos, cuyo objetivo buscará mejorar la productividad al reducir el número de accidentes en las carreteras. La aplicabilidad de la IA en la gestión del transporte en las urbes resulta ser muy prometedor para las autoridades de transporte, ya que les permitirá determinar la forma de gestionar procesos y problemas del sector con una factibilidad por encima de los estudios clásicos, hecho que se podría traducir en servicios de calidad, atenuación de riesgos, mejor productividad de activos vitales y por defecto una mayor aceptación del proceso implementado por la población.

\section{Desafíos de la Inteligencia Artificial en el sector público}

La administración pública
difícilmente puede mantenerse al día
con el rápido desarrollo de la IA, que se
refleja en la falta de programas concretos
de gobernanza y legislación de la IA. Si
bien los desafíos de la IA y los posibles
efectos adversos en la sociedad han
comenzado a llamar la atención de
los investigadores (Wirtz et al, 2020).
Las tendencias y las necesidades
determinadas objetivamente con
respecto a la introducción de la IA en el
sistema y proceso de la administración
pública, se han establecido o reflejado
en muchos documentos conceptuales,


Yolvi Ocaña-Fernández; Luis Alex Valenzuela-Fernández; Miguel Angel Vera-Flores; Raúl Alberto Rengifo-Lozano

Inteligencia artificial aplicada a la gestión pública

doctrinales y programáticos en el campo de la administración pública de Gran Bretaña y otros países de la región europea (Galanos, 2019).

A medida que la IA avanza, las aplicaciones potenciales al trabajo de administración se multiplicarán, a pesar que los gobiernos, a menudo, ralentizan la adopción de nuevas tecnologías (Bullock, 2019; Sharma, Yadav \& Chopra, 2020). Si bien los posibles casos de uso futuro de la IA en el gobierno siguen estando limitados por los recursos gubernamentales, las oportunidades más obvias e inmediatamente beneficiosas son aquellas en las que la IA puede reducir las cargas administrativas, coadyuvando a resolver problemas de asignación de recursos y asumir tareas significativamente complejas (Mehr et al, 2017). Según Nadikattu (2016) la IA desempeñaría la parte más general que incide directamente en hacer que la práctica de las relaciones públicas sean más eficientes y amigables, mejorando así los resultados de una organización.

Según Dwivedi et al, (2019) los impactos de la IA, en la gestión pública, contemplan tres aspectos. Primero, sobre la fuerza laboral del sector público, al delegar la toma de decisiones a la IA; lo cual resultaría una clara amenaza clásica de la sustitución laboral. Segundo, direccionar el incremento de la dinámica en la toma de decisiones públicas respaldada por $\mathrm{IA}$; debido que las aplicaciones de la IA tienden a introducir aspectos no diáfanos al reducir la capacidad de los no expertos para auditar los mecanismos que conducen a los resultados de las decisiones. Tercero, la disminución de cuestiones de opacidad en relación al desempeño de la IA y su asequibilidad con la población; ya que los algoritmos de IA tienden a sugerir que el nivel de transparencia, trazabilidad y explicabilidad son inversamente proporcionales a su complejidad.

En entornos con limitación de recursos, las organizaciones públicas no apuestan por invertir en procesos masivos de actualización del hardware y software empleado; sin embargo, a medida que los costos se hacen más accesibles y la IA gana en replicabilidad, escalabilidad y eficiencia, las mismas presiones que retrasan el salto tecnológico en la gestión pública podrían catalizar que dichas herramientas se conviertan en inversiones atractivas debido a su connotada eficiencia (Etscheid, 2019). De acuerdo con la propuesta de Merh et al, (2019) si alguna entidad pública estuviera interesada en implementar procesos basados en IA pueden aprender de procesos de implementación de IA por parte del sector privado. Además, los citados autores remarcaron que dicha entidad deberá considerar las siguientes estrategias para aplicar la IA a su campo de acción: hacer que la IA forme parte de un programa centrado en el ciudadano y basado en objetivos; obtener la opinión de los ciudadanos; aprovechamiento de los recursos existentes; estar preparado para el tratamiento de datos y cuidando la privacidad; mitigación de riesgos éticos.

Otro aspecto relevante es dado a conocer por Allam \& Dhunny (2019) sobre el progreso de las urbes las que, inevitablemente, se están volcando cada vez más hacia tecnologías especializadas para abordar temas relacionados con la sociedad, la ecología, la morfología y muchos otros. Sobre dicha base emerge el concepto emergente de Smart Cities, lo cual fomenta enormemente esta perspectiva al promover la incorporación de sensores y Big Data a través del denominado 
Internet de las cosas (Ismagilova, Hughes, Dwivedi \& Raman 2019). Esta inevitable oleada de datos trae nuevas posibilidades en el diseño y la gestión de ciudades tanto como perspectivas económicas. El procesamiento de la big data mediada por IA puede contribuir en gran medida al desarrollo del tejido urbano en las dimensiones de sostenibilidad y habitabilidad.

$$
\text { El empleo de la IA por parte }
$$

de entidades del Estado para realizar diversas tareas, podría ser muy relevante, ya que conllevaría a desarrollar una perspectiva más atractiva para su implementación; sin embargo, todavía es imposible hablar de la implementación integrada, totalmente probada y debidamente regulada de este tipo de tecnología por lo tanto, se sugiere profundizar en este tema desde un punto de vista teórico (pronóstico), tomando en cuenta las posibles direcciones y posibilidades en cuanto al uso de dicha tecnología y unidades (Morkhat, et al, 2019; Butcher \& Beridze, 2019).

Según Manyika et al, (2017) existe una relación positiva entre los beneficios y los niveles de adopción de IA en diversos sectores de la economía global, que se traducen en el contexto de una mayor eficiencia, productividad mejorada y confiabilidad. Pero Jarrahi (2018) mencionó que dicha imagen positiva de innovación no se encuentra del todo aceptada ya que las estimaciones para el desplazamiento laboral producto de la introducción de IA o mecanismos equivalentes (big data, machine learning) pronostican que hasta la tercera parte del mercado laboral actual podrían verse seriamente comprometido hacia el 2030

Si bien es cierto que están surgiendo diversas aplicaciones basadas en IA para el sector público, que prometen un gran valor con respecto a la fuerza laboral y la productividad, otro hecho que no puede negar es que ello conlleva desafíos importantes que son cruciales para su implementación y uso exitosos. En tal sentido, Wirtz et al, (2020) observaron desafíos apremiantes respecto a la responsabilidad e implementación de la IA, así como los problemas sociales y éticos que potencialmente amenazan el uso exitoso de la IA y la respectiva creación de valor para el sector público y la sociedad en su conjunto. Si dicho desafío procura un constante crecimiento, tal como viene dándose con el campo de la IA y sus posibilidades, es un hecho que ello procurará una gestación de diversas y novedosas problemáticas que, probablemente, comprometan sectores sensibles de la sociedad y dicho problema tendría a agravarse ya que la IA es una tecnología que trasciende la oquedad del grueso de la población, siendo este aspecto uno de los que catalice lo gravoso de posibles coyunturas de implementación intensiva de la IA en la sociedad

Dado que los nuevos dispositivos tecnológicos basados en IA tienden a reemplazar el trabajo humano a largo plazo, el futuro de la administración pública se ve amenazado, por lo tanto, los gobiernos deben llevar a cabo iniciativas de capacitación de los trabajadores en el uso de dicha tecnología (Shava \& Hofisi, 2017). Según lo mencionado por Bullock (2019) converge la tendencia de que, con las tecnologías actuales, muchas tareas llevadas a cabo por actores humanos ya pueden ser llevadas a cabo de manera más eficiente y efectiva por la IA. Ello sugiere que las tareas realizadas por burócratas humanos, en muchos dominios, pueden ser incrementadas $\mathrm{o}$ automatizadas por IA. A medida que avance el debate digital, varios gobiernos, especialmente 
Yolvi Ocaña-Fernández; Luis Alex Valenzuela-Fernández; Miguel Angel Vera-Flores; Raúl Alberto Rengifo-Lozano

Inteligencia artificial aplicada a la gestión pública

en el mundo en desarrollo, deberían expresar sus preocupaciones en lugar de simplemente permitir que los estados desarrollados dicten el camino del desarrollo hacia ellos. Los Estados deberían poder integrar a las partes interesadas pertinentes, incluida la organización del sector privado, y trazar el camino a seguir en términos de cómo debería adoptarse y en qué medida.

\section{Conclusiones}

La disrupción impulsada por la tecnología se está produciendo a un ritmo y una escala nunca antes vistos en la historia. Oleadas de tecnología, como big data y machine learning las cuales permiten el tratamiento de ingentes volúmenes de data orientado hacia un fin concreto ello debido a que en cada minuto se generan millones de datos que de ser procesados bajo dichas tecnologías aportarían información relevante y pertinente para una adecuada toma de decisiones, están remodelando el modus vivendi a nivel global de manera profunda. Está surgiendo un nuevo mundo en el que muchas de las clases laborales actuales desaparecerán, mientras que surgen otras nuevas que requieren conjuntos de habilidades completamente diferentes.

El mundo está al borde de la tan esperada transformación digital orquestada por la Cuarta Revolución Industrial, la misma que se basa en parte en el desarrollo y aplicaciones de la IA. A pesar de los beneficios percibidos de una mayor eficiencia $y$ eficacia en la prestación de servicios, la cuarta revolución industrial presenta en gran medida numerosos desafíos para la administración pública en los países en desarrollo que carecen de suficientes recursos humanos y materiales para ejecutar los enormes avances tecnológicos consiguientes. Dicho aspecto debe ser entendido desde la realidad de ausencia de expertos en la materia en una cantidad adecuada para que pueda darse un proceso efectivo de implementación, además de tener en cuenta el factor limitante de las economías de la región que muy pocas veces apuestan por la formación técnico-científica de cuadros que lideren dichas posibilidades y puedan trabajar en ello. Quizás este delicado aspecto es uno de los más álgidos que sobrepasan las expectativas, ya que su solución responde a las políticas adoptadas por la gestión de turno.

Es probable que el uso creciente de IA desafíe las normas culturales y actúe como una barrera potencial dentro de ciertos sectores de la población. También se encuentra el riesgo latente de que la IA puede supera el desempeño humano en muchos trabajos y con ello podría, inevitablemente, reemplazarlos. No cabe duda, sobre lo mencionado, que la IA seguirá mejorando su capacidad e infiltrándose en muchos más dominios del quehacer de la sociedad; por ello la preocupación creciente de que la IA usurpe o extinga puestos de trabajo y reemplace a los empleados humanos impedirá generar la confianza de las personas en la IA; pero que, debido a su eficacia, será cuasi inevitable dicha tendencia. De hecho, al ser tendencia y estar presente de una u otra manera en la vida social, es un hecho ineluctable que el viraje hacia procesos mediados por IA en diversos campos y actividades se materialicen, pero la cuestión radical es ¿qué tan preparados estamos al respecto?, y en el caso de acceder a mecanismos y procesos basados en IA, cabe preguntarse ¿existen cuadros profesionales lo suficientemente 
formados como para poder sacar partido de la IA y adaptarla a procesos de desarrollo nacional?; y por otro lado ien el contexto local y asumiendo los niveles de educación de los países de la región y las brechas culturales ¿cómo y cuánto seria el nivel de aceptación/rechazo de la implementación de la IA en la gestión pública por parte de la población? Dichos dilemas, a los cuales no podemos estar exentos, formarán parte de la próxima agenda investigativa respecto del campo de las posibilidades e implicancias de la adecuación de la IA en nuestros países en vías del ansiado desarrollo.

\section{Referencias bibliograficas}

Abbasi, M., \& El Hanandeh, A. (2016). Forecasting municipal solid waste generation using artificial intelligence modelling approaches. Waste management, 56, 13-22. https://doi. org/10.1016/j.wasman.2016.05.018

Abduljabbar, R., Dia, H., Liyanage, S., \& Bagloee, S. A. (2019). Applications of artificial intelligence in transport: An overview. Sustainability, 11(1), 1-24. https://doi.org/10.3390/su11010189.

Allam, Z., \& Dhunny, Z. A. (2019). On big data, artificial intelligence and smart cities. Cities, 89, 80-91. https://doi. org/10.1016/j.cities.2019.01.032.

Agarwal, P. K. (2018). Public administration challenges in the world of $\mathrm{Al}$ and Bots. Public Administration Review, 78(6), 917-921. https://doi. org/10.1111/puar.12979.

Butcher, J., \& Beridze, I. (2019). What is the State of Artificial Intelligence Governance Globally? The RUSI Journal, 164(5-6), 88-96. doi: https:// doi.org/10.1080/03071847.2019.169 4260.

Bullock, J. B. (2019). Artificial intelligence, discretion, and bureaucracy. The American Review of Public Administration, 49(7), 751-761. https://doi. org/10.1177/0275074019856123.

Cath, C., Wachter, S., Mittelstadt, B., Taddeo, M., \& Floridi, L. (2018). Artificial intelligence and the 'good society': the US, EU, and UK approach. Science and engineering ethics, 24(2), 505-528. https://doi. org/10.1007/s11948-017-9901-7.

Chui, M. (2017). Artificial intelligence the next digital frontier?. McKinsey and Company Global Institute, Shanghai: China. https://bit.ly/33TVo6N.

Duan, Y., Edwards, J. S., \& Dwivedi, Y. K. (2019). Artificial intelligence for decision making in the era of Big Data - evolution, challenges and research agenda. International Journal of Information Management, 48, 63-71. https://doi.org/10.1016/j. iiinfomgt.2019.01.021.

Dwivedi, Y. K., Hughes, L., Ismagilova, E., Aarts, G., Coombs, C., Crick, T., ... \& Galanos, V. (2019). Artificial Intelligence (AI): Multidisciplinary perspectives on emerging challenges, opportunities, and agenda for research, practice and policy. International Journal of Information Management, 101994. https://doi. org/10.1016/j.ijinfomgt.2019.08.002.

Etscheid, J. (2019, September). Artificial Intelligence in Public Administration. In International Conference on Electronic Government (pp. 248261). Springer, Cham.

Galanos, V. (2019). Exploring expanding expertise: artificial intelligence as an existential threat and the role of prestigious commentators, 2014-2018. Technology Analysis \& Strategic Management, 31(4), 421432. https://doi.org/10.1080/0953732 $\underline{5.2018 .1518521 .}$ 
Yolvi Ocaña-Fernández; Luis Alex Valenzuela-Fernández; Miguel Angel Vera-Flores; Raúl Alberto Rengifo-Lozano

Inteligencia artificial aplicada a la gestión pública

Galloway, C., \& Swiatek, L. (2018). Public relations and artificial intelligence: It's not (just) about robots. Public relations review, 44(5), 734-740. https://doi.org/10.1016/j. pubrev.2018.10.008.

Haenlein, M., \& Kaplan, A. (2019). A Brief History of Artificial Intelligence: On the Past, Present, and Future of Artificial Intelligence. California Management Review, 000812561986492. https:// doi.org/10.1177/0008125619864925.

Helbing, D., Frey, B. S., Gigerenzer, G., Hafen, E., Hagner, M., Hofstetter, Y., ... \& Zwitter, A. (2019). Will democracy survive big data and artificial intelligence?. In Towards digital enlightenment (pp. 73-98). Springer, Cham.

Henman, P. (2020). Improving public services using artificial intelligence: possibilities, pitfalls, governance. Asia Pacific Journal of Public Administration, 1-13. https://doi.org/1 $0.1080 / 23276665.2020 .1816188$.

Ismagilova, E., Hughes, L., Dwivedi, Y. K., \& Raman, K. R. (2019). Smart cities: Advances in research - An information systems perspective. International Journal of Information Management, 47, 88-100. https://doi. org/10.1016/j.ijinfomgt.2019.01.004.

Issa, H., Sun, T., \& Vasarhelyi, M. A. (2016). Research ideas for artificial intelligence in auditing: The formalization of audit and workforce supplementation. Journal of Emerging Technologies in Accounting, 13(2), 1-20. https://doi. org/10.2308/jeta-10511.

Jarrahi, M. H. (2018). Artificial intelligence and the future of work: Human-Al symbiosis in organizational decision making. Business Horizons, 61(4), 577-586. https://doi.org/10.1016/j. bushor.2018.03.007.
Kouziokas, G. N. (2017). The application of artificial intelligence in public administration for forecasting high crime risk transportation areas in urban environment. Transportation Research Procedia, 24, 467$473 . \quad$ https://doi.org/10.1016/j. trpro.2017.05.083.

Manyika, J., Lund, S., Chui, M., Bughin, J., Woetzel, J., Batra, P., Ko, R. \& Sanghvi, S. (2017). Jobs lost, jobs gained: Workforce transitions in a time of automation. McKinsey Global Institute. http://hdl.voced.edu. au/10707/444873.

Mehr, H., Ash, H., \& Fellow, D. (2017). Artificial intelligence for citizen services and government. Ash Cent. Democr. Gov. Innov. Harvard Kennedy Sch., no. August, 1-12.

Mikhaylov, S. J., Esteve, M., \& Campion, A. (2018). Artificial intelligence for the public sector: opportunities and challenges of cross-sector collaboration. Philosophical Transactions of the Royal Society A: Mathematical, Physical and Engineering Sciences, 376(2128), 1-21. http://dx.doi.org/10.1098/ rsta.2017.0357.

Morkhat, P. M., Ponkin, I. V., Markhgeym, M. V., Botnev, V. K., \& Turganbayev, A. O. (2019). Artificial intelligence versus public administration: limitations of application. Humanities \& Social Sciences Reviews, 7(3), 516-520. https://doi.org/10.18510/ hssr.2019.7376.

Nadikattu, R. R. (2016). The emerging role of artificial intelligence in modern society. International Journal of Creative Research Thoughts, 4(4), 906-911. https://ssrn.com/ abstract $=3652429$.

Park, D. A. (2017). A study on conversational public administration service of the Chatbot based on 
artificial intelligence. Journal of Korea Multimedia Society, 20(8), 1347-1356. https://doi.org/10.9717/ kmms.2017.20.8.1347.

Press, G. (2017). Top 10 hot artificial intelligence (AI) technologies. Forbes, Jan. 23.

Sharma, G. D., Yadav, A., \& Chopra, R. (2020). Artificial intelligence and effective governance: A review, critique and research agenda. Sustainable Futures, 2, 100004. https://doi.org/10.1016/j. sftr.2019.100004.

Shava, E., \& Hofisi, C. (2017). Challenges and opportunities for public administration in the fourth industrial revolution. African Journal of Public Affairs, 9(9), 203-215. https://bit.ly/3ISuyCn.

Sun, T. Q., \& Medaglia, R. (2019). Mapping the challenges of Artificial Intelligence in the public sector: Evidence from public healthcare. Government Information Quarterly, 36(2), 368-383. https://doi. org/10.1016/j.giq.2018.09.008.

Vogl, T. M., Seidelin, C., Ganesh, B.,
\& Bright, J. (2019). Algorithmic Bureaucracy: Managing Competence, Complexity, and Problem Solving in the Age of Artificial Intelligence. SSRN Electronic Journal. https://doi. org/10.2139/ssrn.3327804.

Wirtz, B. W., Weyerer, J. C., \& Geyer, C. (2019). Artificial intelligence and the public sector-applications and challenges. International Journal of Public Administration, 42(7), 596615. https://doi.org/10.1080/0190069 2.2018.1498103.

Wirtz, B. W., Weyerer, J. C., \& Sturm, B. J. (2020). The Dark Sides of Artificial Intelligence: An Integrated Al Governance Framework for Public Administration. International Journal of Public Administration, 43(9), 818829. https://doi.org/10.1080/0190069 2.2020.1749851.

Zheng, Y., Yu, H., Cui, L., Miao, C., Leung, C., \& Yang, Q. (2018). SmartHS: An Al platform for improving government service provision. In 32nd AAAl conference on artificial intelligence. AAAI: Association for the Advancement of Artificial Intelligence, 7704-7712. https://bit.ly/3m4P7eL. 